\title{
Convergence in capacity on compact Kähler manifolds
}

\author{
SŁawomir Dinew And Phạm HoÀng HiệP
}

\begin{abstract}
The aim of this note is to study the convergence in capacity for functions in the class $\mathcal{E}(X, \omega)$. We study the problem under several restrictions on the Monge-Ampère measures of the functions considered, such as common domination by a fixed measure or control on the variation.
\end{abstract}

Mathematics Subject Classification (2010): 32W20 (primary); 32Q15 (secondary).

\section{Introduction}

In $[2,3]$ Bedford and Taylor laid down the foundations of the theory of the complex Monge-Ampère operator which is nowadays a central part of pluripotential theory. In [3] the notion of relative capacity $C_{n}$ was introduced (see Section 2 for the definitions of all the notions appearing in this note). Initially Bedford and Taylor used this capacity to solve deep problems concerning small sets in pluripotential theory. It was soon realized, however, that capacities are very useful technical tools in solving Monge-Ampère equations with singular data. Especially the discovery of Xing [21], who proved that the complex Monge-Ampère operator is continuous with respect to convergence in capacity, attracted much interest. This is in contrast to convergence in $L^{p}, 1<p<\infty$, since it is known [8] that the Monge-Ampère operator is discontinuous with respect to such topology. Recently convergence in capacity in the setting of domains in $\mathbb{C}^{n}$ (which for brewity will be referred to as the local setting in this note) was studied by many authors. We refer to [4-6,9, 15, 17], which is by far an incomplete list of recent contributions, where the reader may obtain a complete picture of the developments in the field.

Quite recently alternative pluripotential theory on compact Kähler manifolds was developed by Guedj and Zeriahi [12,13] and Kołodziej [18]. It should be noted

The first named author was partially supported by Polish ministerial grant N N 201271135 . The final version of the note was finished while the second named author was a post doctoral fellow in CIRM, Trento. He wishes to thank this institution for its hospitality and perfect working conditions.

Received March 24, 2010; accepted May 20, 2011. 
that both theories differ significantly especially when it comes to global results some of the differences were discussed in [13] and [11]. In [18] Kołodziej introduced the capacity $C_{X, \omega}$ on a compact Kähler manifold $(X, \omega)$, which is modelled on the relative capacity $C_{n}$ of Bedford and Taylor from the local setting. Despite its non nocal character this notion plays similar fundamental role as $C_{n}$ does in solving complex Monge-Ampère equations.

In [13] Guedj and Zeriahi (building on local desults due to Cegrell [5,6]) introduced the new Cegrell class $\mathcal{E}(X, \omega)$ of $\omega$-psh functions for which the complex Monge-Ampère operator is well-defined. Roughly speaking, it is the largest class of $\omega$-psh functions where the Monge-Ampère operator behaves like it does in the bounded functions setting. The authors obtained various basic properties of this function class. Later on, building on their work Xing [22] studied the convergence in capacity $C_{X, \omega}$ (one should also mention that various results in the bounded functions setting were also known (compare $[14,19])$ ). As Xing noticed, however, various technical difficulties appear in the analysis in the whole $\mathcal{E}(X, \omega)$ and therefore some of his results are proven only in the smaller class $\mathcal{E}^{1}(X, \omega)$. This leads to the question of what remains true in $\mathcal{E}(X, \omega)$.

The aim of the present note is to answer this question. The main goal will be to study the convergence in capacity $C_{X, \omega}$ in the class $\mathcal{E}(X, \omega)$ - Section 3 . We obtain fairly complete description of that convergence under various additional assumptions such as common domination of the Monge-Ampère measures of the functions by a fixed measure or assumptions on the measures' variation. Analogous results in the local case (except Theorem 3.6 and Lemma 3.7 which are completely new) were obtained by Kołodziej and Cegrell in [9]. We wish to point out that while some of the results look as a straightforward generalizations of the corresponding theorems due to Xing [22], the applied techniques are by necessity of different nature, which allows us to deal with the formerly intractable case of functions in $\mathcal{E}(X, \omega) \backslash \mathcal{E}^{1}(X, \omega)$. These essential new technical tools, namely the partial comparison principle and the uniqueness of solutions to the Monge-Ampère equation in the class $\mathcal{E}(X, \omega)$, are borrowed from [11].

The results obtained, in the authors' opinion, yield better understanding of the class $\mathcal{E}(X, \omega)$ and the non-linear nature of the Monge-Ampère operator in this singular setting.

ACKNOWLEDGEMENTS. The authors would like to thank an anonymous referee of the previous draft of this manuscript whose remarks improved significantly the exposition.

\section{Preliminaries}

First we recall some elements of pluripotential theory that will be used in the paper. We refer the reader to [20] for a recent general overview of pluripotential theory both in the local and in the Kähler setting. 
Given a domain $\Omega$ in $\mathbb{C}^{n}$ one associates to any compact $K \subset \Omega$ the BedfordTaylor relative capacity $C_{n}$ introduced in [3]:

Definition 2.1. Let $K$ be a compact subset of a relatively compact domain $\Omega$ in $\mathbb{C}^{n}$. Then the relative capacity $C_{n}(K, \Omega)$ is defined by

$$
C_{n}(K, \Omega)=\sup \left\{\int_{K}\left(d d^{c} u\right)^{n}: u \in \operatorname{PSH}(\Omega),-1 \leq u \leq 0\right\},
$$

where $\operatorname{PSH}(\Omega)$ denotes the space of plurisubharmonic functions in $\Omega$.

Throughout the note $X$ will denote a (fixed) compact Kähler manifold of complex dimension $n$ equipped with a fundamental form $\omega=\omega_{X}$ normalized by $\int_{X} \omega^{n}=1$.

We shall deal with the $\omega$-plurisubharmonic functions on $X$, which are defined as follows:

Definition 2.2. An upper semicontinuous function $\varphi: X \rightarrow[-\infty,+\infty)$ is called $\omega$-plurisubharmonic ( $\omega$-psh for short) if $\varphi \in L^{1}(X)$ and $\omega_{\varphi}:=\omega+d d^{c} \varphi \geq 0$, where the inequality is understood in the sense of currents. By $\operatorname{PSH}(X, \omega)$ (respectively $\left.\operatorname{PSH}^{-}(X, \omega)\right)$ we denote the set of $\omega$-psh (respectively negative $\omega$-psh) functions on $X$.

Modelling on the definition of $C_{n}$ above Kołodziej in [18] introduced the capacity $C_{X, \omega}$ :

Definition 2.3. Given any Borel set $E \subset X$ we define its capacity $C_{X, \omega}$ on $X$ as

$$
C_{X}(E)=C_{X, \omega}(E)=\sup \left\{\int_{E} \omega_{\varphi}^{n}: \varphi \in \operatorname{PSH}(X, \omega),-1 \leq \varphi \leq 0\right\},
$$

where $\omega_{\varphi}^{n}=\left(\omega+d d^{c} \varphi\right)^{n}$ and $n=\operatorname{dim} X$.

We refer to $[12,18]$ for more information about this capacity.

It follows from the locality of the Monge-Ampère operator that it can be defined for any bounded $\omega$-psh function [3]. As noticed by Guedj and Zeriahi this is not so for unbounded ones and therefore one has to restrict to a subclass of $\operatorname{PSH}(X, \omega)$. The following class of $\omega$-psh fuctions was introduced by Guedj and Zeriahi in [13]:

\section{Definition 2.4.}

$$
\mathcal{E}(X, \omega)=\left\{\varphi \in \operatorname{PSH}(X, \omega): \lim _{j \rightarrow \infty} \int_{\{\varphi>-j\}} \omega_{\max (\varphi,-j)}^{n}=\int_{X} \omega^{n}=1\right\} .
$$

Intuitively the space $\mathcal{E}(X, \omega)$ consists of those functions which have mild singularities (or do not have $-\infty$ poles at all), so that no Monge-Ampère mass concentrates near those poles. In particular it can be proven (see [13]) that any such function must have zero Lelong numbers everywhere. 
Let us also define

$$
\mathcal{E}^{-}(X, \omega)=\mathcal{E}(X, \omega) \cap P S H^{-}(X, \omega) .
$$

We refer to [13], where the reader can find the basic properties of these function classes.

The convergence in capacity is defined as follows:

Definition 2.5. Let $u_{j}, u \in \operatorname{PSH}(X, \omega)$. We say that $\left\{u_{j}\right\}$ converges to $u$ in $C_{X}$ if

$$
C_{X}\left(\left\{\left|u_{j}-u\right|>\delta\right\}\right) \rightarrow 0
$$

as $j \rightarrow \infty$, for every (fixed) $\delta>0$.

For our later reference we shall need the notion of uniform absolute continuity with respect to capacity. It is modelled on the uniform continuity with respect to a measure.

Definition 2.6. A family of positive measures $\left\{\mu_{\alpha}\right\}$ on $X$ is said to be uniformly absolutely continuous with respect to $C_{X}$-capacity if for every $\epsilon>0$ there exists $\delta>0$ such that for each Borel subset $E \subset X$ satisfying $C_{X}(E)<\delta$ the inequality $\mu_{\alpha}(E)<\epsilon$ holds for all $\alpha$. We denote this by $\mu_{\alpha} \ll C_{X}$ uniformly for $\alpha$.

Note that by [12, Theorem 6.2] we know that the sets satisfying $C_{X}(E)=0$ are precisely the $\omega$-pluripolar sets. In particular all measures that are continuous with respect to capacity must vanish on pluripolar sets.

The next two propositions are well-known:

Proposition 2.7 (cf. [13, Theorem 1.5]). Let $u \in \mathcal{E}(X, \omega), v \in \operatorname{PSH}(X, \omega)$. Then

$$
\int_{\{u \leq v\}} \omega_{\max (u, v)}^{k} \wedge T=\int_{\{u \leq v\}} \omega_{u}^{k} \wedge T,
$$

for all $1 \leq k \leq n$ and $T=\omega_{\varphi_{1}} \wedge \ldots \wedge \omega_{\varphi_{n-k}}$ with $\varphi_{1}, \ldots, \varphi_{n-k} \in \mathcal{E}(X, \omega)$.

Proposition 2.8 (cf. Proposition 3.6 in [12]). Let $u \in \mathrm{PSH}^{-}(X, \omega)$. Then for $t \geq 0$

$$
C_{X}(\{u<-t\}) \leq \frac{\left|\sup _{X} u\right|+c}{t},
$$

where the positive constant $c$ does not depend on $u$.

Next we state a simple criterion for uniform continuity in terms of capacity.

Proposition 2.9. Let $u_{j} \in \mathcal{E}^{-}(X, \omega)$. Then the following two statements are equivalent:

i) $\inf _{j \geq 1} \sup _{X} u_{j}>-\infty$ and $\omega_{u_{j}}^{n} \ll C_{X}$ uniformly for $j \geq 1$;

ii) $\lim _{t \rightarrow+\infty} \overline{\lim }_{j \rightarrow \infty} \int_{\left\{u_{j} \leq-t\right\}} \omega_{u_{j}}^{n}=0$. 
Proof. i) $\Rightarrow$ ii) This is a direct application of Proposition 2.8.

ii) $\Rightarrow$ i) We claim that $\inf _{j \geq 1} \sup _{X} u_{j}>-\infty$.

Suppose on contrary that $\inf _{j \geq 1} \sup _{X} u_{j}=-\infty$. Hence

$$
\varlimsup_{j \rightarrow \infty} \int_{\left\{u_{j} \leq-t\right\}} \omega_{u_{j}}^{n}=\varlimsup_{j \rightarrow \infty} \int_{X} \omega_{u_{j}}^{n}=1,
$$

but this contradicts the assumption that

$$
\lim _{t \rightarrow+\infty} \varlimsup_{j \rightarrow \infty} \int_{\left\{u_{j} \leq-t\right\}} \omega_{u_{j}}^{n}=0 .
$$

Let us fix $\epsilon>0$. We choose $t_{0}$ and then $j_{0}=j_{0}\left(t_{0}\right)>1$ such that

$$
\int_{\left\{u_{j} \leq-t_{0}\right\}} \omega_{u_{j}}^{n}<\epsilon,
$$

for all $j \geq j_{0}$. For each Borel set $E \subset X$ can estimate

$$
\begin{aligned}
\int_{E} \omega_{u_{j}}^{n} & =\int_{E \cap\left\{u_{j} \leq-t_{0}\right\}} \omega_{u_{j}}^{n}+\int_{E \cap\left\{u_{j}>-t_{0}\right\}} \omega_{u_{j}}^{n} \\
& \leq \int_{\left\{u_{j} \leq-t_{0}\right\}} \omega_{u_{j}}^{n}+\int_{E \cap\left\{u_{j}>-t_{0}\right\}} \omega_{\max \left(u_{j},-t_{0}\right)}^{n} \\
& \leq \int_{\left\{u_{j} \leq-t_{0}\right\}} \omega_{u_{j}}^{n}+t_{0}^{n} C_{X}(E) \leq \epsilon+t_{0}^{n} C_{X}(E)
\end{aligned}
$$

for all $j \geq j_{0}$. On the other hand, since $\sum_{k=1}^{j_{0}} \omega_{u_{k}}^{n} \ll C_{X}$ we can choose $\delta_{1}>0$ such that $\sum_{k=1}^{j_{0}} \omega_{u_{k}}^{n}(E)<\epsilon$ for all Borel sets $E \subset X$ with $C_{X}(E)<\delta_{1}$. Hence $\omega_{u_{j}}^{n}(E)<2 \epsilon$ for all $j \geq 1$ and all Borel sets $E \subset X$, such that $C_{X}(E)<\delta=$ $\min \left(\delta_{1}, \frac{\epsilon}{t_{0}^{n}}\right)$.

The next proposition is a modified version of [22, Lemma 2]:

Proposition 2.10. Let $u_{j}, v_{j} \in \mathcal{E}^{-}(X, \omega)$ be such that $u_{j} \geq v_{j}$ for $j \geq 1$. Assume that $\omega_{v_{j}}^{n} \ll C_{X}$ uniformly for $j \geq 1$. Let also $\inf _{j \geq 1} \sup _{X} v_{j}>-\infty$. Then $\omega_{u_{j}}^{n} \ll C_{X}$ uniformly for $j \geq 1$.

Proof. By [13, Theorem 1.5] we have

$$
\int_{\left\{u_{j}<-2 t\right\}} \omega_{u_{j}}^{n} \leq 2^{n} \int_{\left\{v_{j}<\frac{u_{j}}{2}-t\right\}} \omega_{\frac{u_{j}}{2}}^{n} \leq 2^{n} \int_{\left\{v_{j}<\frac{u_{j}}{2}-t\right\}} \omega_{v_{j}}^{n} \leq 2^{n} \int_{\left\{v_{j}<-t\right\}} \omega_{v_{j}}^{n},
$$

for every $t>0$. By Proposition 2.9 we obtain $\omega_{u_{j}}^{n} \ll C_{X}$ uniformly for $j \geq 1$. 
We remark that the proof shows that the result still holds for any family of functions $u_{\alpha}$ and $v_{\alpha}, \alpha \in \Lambda$ instead of just sequences.

Proposition 2.11. Let $u_{j} \in \mathcal{E}(X, \omega), u \in P S H(X, \omega)$ be such that $u_{j} \rightarrow u$ in $C_{X}$. Then the following two statements are equivalent:

i) $u \in \mathcal{E}(X, \omega)$;

ii) $\omega_{u_{j}}^{n} \ll C_{X}$ uniformly for $j \geq 1$.

Proof. i) $\Rightarrow$ ii) By Proposition 2.7 we have

$$
\begin{aligned}
\int_{\left\{u_{j} \leq-t\right\}} \omega_{u_{j}}^{n} & =\int_{\left\{u_{j} \leq-t\right\}} \omega_{\max \left(u_{j},-t\right)}^{n} \leq \int_{\{u \leq-t+1\}} \omega_{\max \left(u_{j},-t\right)}^{n}+\int_{\left\{\left|u_{j}-u\right|>1\right\}} \omega_{\max \left(u_{j},-t\right)}^{n} \\
& \leq \int_{\{u \leq-t+1\}} \omega_{\max \left(u_{j},-t\right)}^{n}+t^{n} C_{X}\left(\left\{\left|u_{j}-u\right|>1\right\}\right) .
\end{aligned}
$$

By coupling the quasi-continuity of $u$ (in [3, Theorem 3.5]) and the facts that $\omega_{\max \left(u_{j},-t\right)}^{n} \ll C_{X}$ uniformly for $j \geq 1$ and $\omega_{\max (u,-t)}^{n} \ll C_{X}$ uniformly for $t>0$ (see Proposition 2.10), we get

$$
\varlimsup_{t \rightarrow+\infty} \varlimsup_{j \rightarrow \infty} \int_{\left\{u_{j} \leq-t\right\}} \omega_{u_{j}}^{n} \leq \varlimsup_{t \rightarrow+\infty} \int_{\{u \leq-t+1\}} \omega_{\max (u,-t)}^{n}=0 .
$$

By Proposition 2.9 we obtain $\omega_{u_{j}}^{n} \ll C_{X}$ uniformly for $j \geq 1$.

ii) $\Rightarrow$ i) This is a part in [22, Theorem 3].

Finally we would like to mention that recently Benelkourchi, Guedj and Zeriahi [1] have developed analogous theory for compact complex manifolds equipped with big forms (i.e. smooth semi-positive forms $\omega$ satisfying $\int_{X} \omega^{n}>0$ ). We leave to the reader as an exercise to check that our results still hold in this more general setting.

\section{Convergence in capacity}

Below we prove various results regarding convergence in capacity in the class $\mathcal{E}(X, \omega)$. We begin with a technical theorem which will be used later on. This result is a generalization of similar ideas in [14, Lemma 2.3] (where the case of bounded functions is considered) and in [22, Theorem 2], where the author works in the smaller function class $\mathcal{E}^{1}(X, \omega)$.

Theorem 3.1. Let $u_{j}, v_{j} \in \mathcal{E}(X, \omega)$ be such that

i) $\omega_{u_{j}}^{n} \ll C_{X}$ uniformly for $j \geq 1$;

ii) $\lim _{j \rightarrow \infty} \int_{\left\{u_{j}<v_{j}-\delta\right\}} \omega_{u_{j}}^{n}=0$ for all $\delta>0$.

Then $\lim _{j \rightarrow \infty} C_{X}\left(\left\{u_{j}<v_{j}-\delta\right\}\right)=0$ for all $\delta>0$. 
Proof. We can assume, by adding constants to both $u_{j}$ and $v_{j}$ if necessary, that $\sup _{X} u_{j}=0$ for all $j \geq 1$. Set $u_{j t}:=\max \left(u_{j},-t\right)$. For each $k=0, \ldots, n$ we will prove inductively that for every $\delta>0$

$$
\lim _{j \rightarrow \infty} \sup \left\{\int_{\left\{u_{j}<v_{j}-\delta\right\}} \omega_{u_{j}}^{n-k} \wedge \omega_{\varphi}^{k}: \varphi \in \operatorname{PSH}(X, \omega),-1 \leq \varphi \leq 0\right\}=0 .
$$

Note that if $k=0$ then (3.1) holds by assumption.

Assume that (3.1) holds for $k-1$. We will prove that

$$
\lim _{j \rightarrow \infty} \sup \left\{\int_{\left\{u_{j}<v_{j}-3 \delta\right\}} \omega_{u_{j}}^{n-k} \wedge \omega_{\varphi}^{k}: \varphi \in \operatorname{PSH}(X, \omega),-1 \leq \varphi \leq 0\right\}=0
$$

for any $\delta>0$. We fix $t \geq 1$ and $\varphi \in \operatorname{PSH}(X, \omega)$ satisfying $-1 \leq \varphi \leq 0$. For notational ease we denote the set $\left\{u_{j}+\frac{\delta}{t} u_{j t}<v_{j}+\frac{\delta}{t} \varphi-2 \delta\right\}$ by $U$ and let also $\eta_{j}, \beta_{j}$ be the $\omega$-psh functions defined respectively by $\eta_{j}:=\frac{v_{j}+\frac{\delta}{t} \varphi-2 \delta}{1+\frac{\delta}{t}}, \beta_{j}:=$ $\frac{u_{j}+\frac{\delta}{t} u_{j t}}{1+\frac{\delta}{t}}$. Then we have

$$
\int_{\left\{u_{j}<v_{j}-3 \delta\right\}} \omega_{u_{j}}^{n-k} \wedge \omega_{\varphi}^{k} \leq \int_{U} \omega_{u_{j}}^{n-k} \wedge \omega_{\varphi}^{k}
$$

since $\frac{\delta \varphi-\delta u_{j t}}{t}>-\delta$.

The measure on the right-hand side can be enlarged to

$$
\frac{t+\delta}{\delta} \omega_{\eta_{j}} \wedge \omega_{u_{j}}^{n-k} \wedge \omega_{\varphi}^{k-1}
$$

Thus, by consecutive application of [11, Theorem 2.3] and yet another set inclusion obtained as the one above we get

$$
\begin{aligned}
& \frac{t+\delta}{\delta} \int_{U} \omega_{\eta_{j}} \wedge \omega_{u_{j}}^{n-k} \wedge \omega_{\varphi}^{k-1} \leq \frac{t+\delta}{\delta} \int_{U} \omega_{\beta_{j}} \wedge \omega_{u_{j}}^{n-k} \wedge \omega_{\varphi}^{k-1} \\
& \leq \frac{t}{\delta} \int_{\left\{u_{j}<v_{j}-\delta\right\}} \omega_{u_{j}}^{n-k+1} \wedge \omega_{\varphi}^{k-1}+\int_{\left\{u_{j}<v_{j}-\delta\right\}} \omega_{u_{j t}} \wedge \omega_{u_{j}}^{n-k} \wedge \omega_{\varphi}^{k-1} .
\end{aligned}
$$

Using form [13, Corollary 1.7 ] this last sum can be in turn estimated by

$$
\begin{aligned}
& \frac{t+\delta}{\delta} \int_{\left\{u_{j}<v_{j}-\delta\right\}} \omega_{u_{j}}^{n-k+1} \wedge \omega_{\varphi}^{k-1}+\int_{\left\{u_{j} \leq-t\right\}} \omega_{u_{j t}} \wedge \omega_{u_{j}}^{n-k} \wedge \omega_{\varphi}^{k-1} \\
& \leq \frac{t+\delta}{\delta} \int_{\left\{u_{j}<v_{j}-\delta\right\}} \omega_{u_{j}}^{n-k+1} \wedge \omega_{\varphi}^{k-1}+\int_{\left\{u_{j} \leq-t\right\}} \omega_{u_{j}}^{n-k+1} \wedge \omega_{\varphi}^{k-1} .
\end{aligned}
$$


By the induction hypothesis we get

$$
\begin{aligned}
& \varlimsup_{j \rightarrow \infty} \sup \left\{\int_{\left\{u_{j}<v_{j}-3 \delta\right\}} \omega_{u_{j}}^{n-k} \wedge \omega_{\varphi}^{k}: \varphi \in \operatorname{PSH}(X, \omega),-1 \leq \varphi \leq 0\right\} \\
& \leq \sup \left\{\int_{\left\{u_{j} \leq-t\right\}} \omega_{u_{j}}^{n-k+1} \wedge \omega_{\varphi}^{k-1}: \varphi \in \operatorname{PSH}(X, \omega),-1 \leq \varphi \leq 0, j \geq 1\right\},
\end{aligned}
$$

for every $t \geq 1$.

Note that $\frac{u_{j}+\varphi}{2} \geq u_{j}-1$, thus by Proposition 2.10 we get that $\omega_{\frac{u_{j}+\varphi}{2}}^{n} \ll C_{X}$ uniformly for $j \geq 1$. Expanding the left-hand side we obtain

$$
\omega_{u_{j}}^{n-k+1} \wedge \omega_{\varphi}^{k-1} \ll C_{X} \text { uniformly for } j \geq 1
$$

Now by letting $t \rightarrow+\infty$ (and using $\omega_{u_{j}}^{n-k+1} \wedge \omega_{\varphi}^{k-1} \ll C_{X}$ uniformly for $j \geq 1$ ), from Proposition 2.8 we obtain

$$
\lim _{j \rightarrow \infty} \sup \left\{\int_{\left\{u_{j}<v_{j}-3 \delta\right\}} \omega_{u_{j}}^{n-k} \wedge \omega_{\varphi}^{k}: \varphi \in \operatorname{PSH}(X, \omega),-1 \leq \varphi \leq 0\right\}=0 .
$$

Theorem 3.1 has various consequences regarding continuity of the Monge-Ampère operator. Below we list some of them.

As a direct corollary we obtain the following theorem:

Theorem 3.2. Let $u_{j}, v_{j} \in \mathcal{E}(X, \omega)$ be such that

i) $\omega_{u_{j}}^{n}+\omega_{v_{j}}^{n} \ll C_{X}$ uniformly for $j \geq 1$;

ii) $\lim _{j \rightarrow \infty}\left[\int_{\left\{u_{j}<v_{j}-\delta\right\}} \omega_{u_{j}}^{n}+\int_{\left\{v_{j}<u_{j}-\delta\right\}} \omega_{v_{j}}^{n}\right]=0$ for all $\delta>0$.

Then $u_{j}-v_{j} \rightarrow 0$ in $C_{X}$.

Recently the second named author obtained a characterization of convergence in capacity for bounded $\omega$-psh functions (in [14, Theorem 2.1]). Our next result provides such a characterization in the class $\mathcal{E}(X, \omega)$ :

Theorem 3.3. Let $u_{j} \in \mathcal{E}(X, \omega)$ and $u \in P S H(X, \omega)$. Then the following three statements are equivalent:

i) $u \in \mathcal{E}(X, \omega)$ and $u_{j} \rightarrow u$ in $C_{X}$;

ii) $\omega_{u_{j}}^{n} \ll C_{X}$ uniformly for $j \geq 1$ and $u_{j} \rightarrow u$ in $C_{X}$;

iii) $\omega_{u_{j}}^{n} \ll C_{X}$ uniformly for $j \geq 1, \varlimsup_{j \rightarrow \infty} u_{j} \leq u$ and $\lim _{j \rightarrow \infty} \int_{\left\{u_{j}<u-\delta\right\}} \omega_{u_{j}}^{n}=$ 0 for all $\delta>0$. 
Proof. i) $\Leftrightarrow$ ii) This equivalence is the content of Proposition 2.11.

ii) $\Rightarrow$ iii) This implication is trivial.

iii) $\Rightarrow$ i) This is a direct application Theorem 3.1, coupled with Proposition 2.11.

Recall that the weak convergence of Monge-Ampère measures does not imply the weak convergence of the corresponding functions (and vice versa). Thus these convergence problems are considered with additional restrictions. Usually results in this direction are called stability theorems in the literature, since they show that suitable small modification of the Monge-Ampère measures do not lead to large deviation of solutions (i.e. the solutions are stable). In [9] good convergence properties were obtained (in the setting of domains in $\mathbb{C}^{n}$ ) under the assumption that all the Monge-Ampère measures are dominated by a fixed measure vanishing on pluripolar sets. In the Kähler setting the corresponding problem was studied by Xing in [22]. He worked in the subclass $\mathcal{E}^{1}(X, \omega) \subset \mathcal{E}(X, \omega)$, however all his arguments can be applied in the whole $\mathcal{E}(X, \omega)$ provided one has uniqueness (modulo an additive constant) for the solutions of the Monge-Ampère equation

$$
\omega_{\varphi}^{n}=\mu, \varphi \in \mathcal{E}(X, \omega),
$$

where $\mu$ is any positive Borel measure satisfying $\int_{X} d \mu=\int_{X} \omega^{n}$ and vanishing on pluripolar sets. This uniqueness statement was recently obtained in [11]. Thus coupling Xing's arguments with Theorem 3.2 one gets the following stability theorem:

Theorem 3.4. Let $u_{j} \in \mathcal{E}(X, \omega)$ and $u \in \operatorname{PSH}(X, \omega)$. Assume that $\omega_{u_{j}}^{n} \leq d \mu$ for some measure $d \mu \ll C_{X}$. Let also $\sup _{X} u_{j}=\sup _{X} u$. Then the following three statements are equivalent:

i) $u_{j} \rightarrow u$ in $C_{X}$.

ii) $u_{j} \rightarrow u$ in $L^{1}$.

iii) $\omega_{u_{j}}^{n} \rightarrow \omega_{u}^{n}$ weakly.

Proof. The implications ii) $\Leftrightarrow$ iii) are essentially due to Xing. In particular in [22, Corollary 1] (and Proposition 2.11) yields ii) $\Rightarrow$ iii). For the other implication one can proceed exactly as in [22, Theorem 8]: below we sketch the details from [22] for the reader's convenience.

Due to the normalization assumption one can extract a subsequence from $u_{j}$ convergent in $L^{1}$ to some function $v \in \operatorname{PSH}(X, \omega)$. Note that one also has $\sup _{X} v=$ $\sup _{X} u$. The result follows if one can show that $v$ actually coincides with $u$, since the chosen convergent subsequence is arbitrary. But the Monge-Ampère measures of the functions in the subsequence are again dominated by $\mu$, hence from [22, Theorem 4] one concludes that $v$ belongs to $\mathcal{E}(X, \omega)$ and moreover the MongeAmpère measures tend weakly to $\omega_{v}^{n}$. Thus one gets $\omega_{u}^{n}=\omega_{v}^{n}$, so by uniqueness (now in $\mathcal{E}(X, \omega)$ ) and normalization $u=v$. Thus, since any subsequence has subsequence convergent in $L^{1}$ to $u$ one concludes that $u_{j} \rightarrow u$ in $L^{1}$. 
Since implication i) $\Rightarrow$ ii) is trivial, we only have to show that ii) implies i). But we know that $\omega_{u_{j}}^{n} \ll C_{X}$ (since all the measures are dominated by $\mu$ ). By [7] (see also in [9, Lemma 1.4]) we know that

$$
\forall M \in \mathbb{R} \lim _{j \rightarrow \infty} \int_{X} \max \left(u_{j}, M\right)-\max (u, M) d \mu=0 .
$$

(Indeed, the results in [7] and [9] are proven in the local case, but this convergence statement is of purely local nature and hence can be easily transplanted to the Kähler setting).

Thus we obtain

$\int_{\left\{u_{j}<u-\delta\right\}} \omega_{u_{j}}^{n}+\int_{\left\{u<u_{j}-\delta\right\}} \omega_{u}^{n} \leq \int_{\left\{u_{j}<M\right\} \cup\{u<M\}} d \mu+\frac{1}{\delta} \int_{X}\left|\max \left(u_{j}, M\right)-\max (u, M)\right| d \mu$.

If $M$ is sufficiently negative by the assumption $\mu \ll C_{X}$ and Proposition 2.8 we obtain that the first term on the right-hand side can be made arbitrarily small (independently of $j$ ). Finally we obtain

$$
\lim _{j \rightarrow \infty}\left[\int_{\left\{u_{j}<u-\delta\right\}} \omega_{u_{j}}^{n}+\int_{\left\{u<u_{j}-\delta\right\}} \omega_{u}^{n}\right]=0 .
$$

Thus by Theorem $3.2 u_{j} \rightarrow u$ in $C_{X}$.

Before we proceed further we recall the notion of variation of a measure. Given any Borel measure $\mu$ (non-necessarily positive) one defines its variation $\|\mu\|$ on a Borel set $A$ by

$$
\|\mu\|(A):=\sup \left\{\sum_{k=1}^{\infty}\left|\mu\left(R_{k}\right)\right|\right\},
$$

where $R_{k}, k \in \mathbb{N}$ is any disjoint at most countable partition of the set $A$ by Borel sets. The classical Hahn decomposition theorem states that in fact that it is enough to consider only the special partition given by the two Hahn sets - intutively - the pieces where $\mu$ is positive and negative.

Remark 3.5. It would be interesting to know whether the above result still holds if instead of domination by a fixed measure one assumes merely that $\omega_{u_{j}}^{n} \ll C_{X}$. Indeed large parts of Xing's proof can be dealt verbatim, yet some details remain unclear to us.

Our next result concerns a new type of stability. Instead of controlling the deviation of $u_{j}$ from $v_{j}$ we impose an assumption on the variation of their MongeAmpère measures. Since in concrete situations the Monge-Ampère measures of functions in concern are given (while the information on the functions themselves is very limited) this result seems more suitable for applications than Theorem 3.2. Such a theorem is new even for bounded $\omega$-psh functions. 
Theorem 3.6. Let $u_{j}, v_{j} \in \mathcal{E}(X, \omega)$ be such that

i) $\lim _{j \rightarrow \infty}\left|\sup _{X} u_{j}-\sup _{X} v_{j}\right|=0$;

ii) $\omega_{u_{j}}^{n}+\omega_{v_{j}}^{n} \ll C_{X}$ uniformly for $j \geq 1$;

iii) $\lim _{j \rightarrow \infty} \int_{X}\left\|\omega_{u_{j}}^{n}-\omega_{v_{j}}^{n}\right\|=0$.

Then $u_{j}-v_{j} \rightarrow 0$ in $C_{X}$.

Note that the first assumption is an obvious normalization which can always be assumed after adding suitable constants to the functions in concern. Before we start the proof we state an auxiliary lemma which might be of independent interest.

Lemma 3.7. Let $u, v \in \mathcal{E}(X, \omega)$. Then for any $k \in\{0, \cdots, n\}$ we have the estimate

$$
\int_{X}\left\|\omega_{u}^{k} \wedge \omega_{v}^{n-k}-\omega_{u}^{n}\right\| \leq 2\left[\int_{X}\left\|\omega_{u}^{n}-\omega_{v}^{n}\right\|\right]^{\frac{n-k}{n}} .
$$

Proof. Set

$$
\mu=\frac{1}{2}\left[\omega_{u}^{n}+\omega_{v}^{n}\right] .
$$

We choose $f, g \in L^{1}(d \mu), f, g \geq 0$ such that

$$
\omega_{u}^{n}=f d \mu, \omega_{v}^{n}=g d \mu .
$$

By the inequality for mixed Monge-Ampère measures ( [10], see also in [11, Theorem 2.1]) and the Hölder inequality we get

$$
\begin{aligned}
& \int_{E} \omega_{u}^{k} \wedge \omega_{v}^{n-k}-\int_{E} \omega_{u}^{n} \geq \int_{E} f^{\frac{k}{n}} g^{\frac{n-k}{n}} d \mu-\int_{E} f d \mu \\
& =\int_{E} f^{\frac{k}{n}}\left[g^{\frac{n-k}{n}}-f^{\frac{n-k}{n}}\right] d \mu \geq-\int_{E} f^{\frac{k}{n}}\left|g^{\frac{n-k}{n}}-f^{\frac{n-k}{n}}\right| d \mu \\
& \geq-\int_{E} f^{\frac{k}{n}}|g-f|^{\frac{n-k}{n}} d \mu \geq-\left[\int_{E} f d \mu\right]^{\frac{k}{n}}\left[\int_{E}|g-f| d \mu\right]^{\frac{n-k}{n}} \\
& \geq-\left[\int_{E}|g-f| d \mu\right]^{\frac{n-k}{n}} \geq-\left[\int_{X}|| \omega_{u}^{n}-\omega_{v}^{n}||\right]^{\frac{n-k}{n}}
\end{aligned}
$$

for any Borel set $E \subset X$. Similarly we get

$$
\int_{X \backslash E} \omega_{u}^{k} \wedge \omega_{v}^{n-k}-\int_{X \backslash E} \omega_{u}^{n} \geq-\left[\int_{X}\left\|\omega_{u}^{n}-\omega_{v}^{n}\right\|\right]^{\frac{n-k}{n}} .
$$

Hence

$$
\int_{E} \omega_{u}^{k} \wedge \omega_{v}^{n-k}-\int_{E} \omega_{u}^{n} \leq\left[\int_{X}\left\|\omega_{u}^{n}-\omega_{v}^{n}\right\|\right]^{\frac{n-k}{n}}
$$


for all Borel sets $E \subset X$. A combination of these inequalities yields

$$
\left|\int_{E} \omega_{u}^{k} \wedge \omega_{v}^{n-k}-\int_{E} \omega_{u}^{n}\right| \leq\left[\int_{X}\left\|\omega_{u}^{n}-\omega_{v}^{n}\right\|\right]^{\frac{n-k}{n}},
$$

for all Borel $E \subset X$. This implies that

$$
\int_{X}\left\|\omega_{u}^{k} \wedge \omega_{v}^{n-k}-\omega_{u}^{n}\right\| \leq 2\left[\int_{X}\left\|\omega_{u}^{n}-\omega_{v}^{n}\right\|\right]^{\frac{n-k}{n}} .
$$

Proof of Theorem 3.6. Our strategy will be to start with the bounded functions case. It will turn out that the general case follows in a rather simple manner from this one.

Case I: $u_{j}, v_{j} \in \operatorname{PSH}(X, \omega) \cap \mathrm{L}^{\infty}(X)$. Set

$$
a_{j}:=\left[\sup \left\{\int_{X}\left\|\omega_{u_{j}}^{k} \wedge \omega_{v_{j}}^{n-k}-\omega_{u_{j}}^{m} \wedge \omega_{v_{j}}^{n-m}\right\|: 1 \leq k, m \leq n\right\}\right]^{\frac{1}{4}} .
$$

Note that our assumptions coupled with Lemma 3.7 yield $\lim _{j \rightarrow \infty} a_{j}=0$. From now on we assume that $a_{j}$ 's are small.

The crucial point in the proof will be the following equality

$$
\lim _{j \rightarrow \infty} \sup _{t \in \mathbb{R}}\left\{\int_{\left\{\left|u_{j}-v_{j}-t\right| \leq a_{j}\right\}} \omega_{u_{j}}^{n}\right\}=1 .
$$

Indeed, suppose (3.2) is already proved. Then we can choose $k_{j} \in \mathbb{R}$ such that

$$
\lim _{j \rightarrow \infty} \int_{\left\{\left|u_{j}-v_{j}-k_{j}\right|>a_{j}\right\}} \omega_{u_{j}}^{n}=0 .
$$

Moreover from iii) we get

$$
\lim _{j \rightarrow \infty} \int_{\left\{\left|u_{j}-v_{j}-k_{j}\right|>a_{j}\right\}} \omega_{v_{j}}^{n}=0 .
$$

By Theorem 3.2 we obtain $u_{j}-v_{j}-k_{j} \rightarrow 0$ in $C_{X}$. Also from i) we get $\lim _{j \rightarrow \infty} k_{j}=0$. Hence $u_{j}-v_{j} \rightarrow 0$ in $C_{X}$.

Thus we have to prove (3.2). Suppose on contrary that there exists $\epsilon_{0}>0$ such that

$$
\int_{\left\{\left|u_{j}-v_{j}-t\right| \leq a_{j}\right\}} \omega_{u_{j}}^{n} \leq 1-2 \epsilon_{0}
$$

for all $t \in \mathbb{R}, j \geq 1$. Set

$$
t_{j}:=\sup \left\{t \in \mathbb{R}: \int_{\left\{u_{j}<v_{j}+t+a_{j}\right\}} \omega_{u_{j}}^{n} \leq 1-\epsilon_{0}\right\} .
$$


Replacing $v_{j}+t_{j}$ by $v_{j}$ we can assume that $t_{j}=0$. Then $\int_{\left\{u_{j}<v_{j}+a_{j}\right\}} \omega_{u_{j}}^{n} \leq 1-\epsilon_{0}$ and $\int_{\left\{u_{j} \leq v_{j}+a_{j}\right\}} \omega_{u_{j}}^{n} \geq 1-\epsilon_{0}$. Hence

$$
\begin{aligned}
\int_{\left\{v_{j}<u_{j}+a_{j}\right\}} \omega_{u_{j}}^{n}= & 1-\int_{\left\{u_{j}+a_{j} \leq v_{j}\right\}} \omega_{u_{j}}^{n}=1-\int_{\left\{u_{j} \leq v_{j}+a_{j}\right\}} \omega_{u_{j}}^{n} \\
& +\int_{\left\{v_{j}-a_{j}<u_{j} \leq v_{j}+a_{j}\right\}} \omega_{u_{j}}^{n} \leq 1-\epsilon_{0} .
\end{aligned}
$$

Consider now the interval $\left[-a_{j}+a_{j}^{2}, a_{j}-a_{j}^{2}\right]$. It contains $\left[1 / a_{j}\right]-2$ disjoint closed intervals each with length $2 a_{j}^{2}$, ([x] denotes the integral part of $x$ ). Observe that the sets $\left\{\left|u_{j}-v_{j}-p\right|<a_{j}^{2}\right\}$ are pairwise disjoint when $p$ varies among the centers of those intervals. Since $\int_{\left\{\left|u_{j}-v_{j}\right| \leq a_{j}\right\}} \omega_{u_{j}}^{n} \leq 1$ we can thus choose $s_{j} \in\left[-a_{j}+a_{j}^{2}, a_{j}-a_{j}^{2}\right]$ satisfying

$$
\int_{\left\{\left|u_{j}-v_{j}-s_{j}\right|<a_{j}^{2}\right\}} \omega_{u_{j}}^{n} \leq 2 a_{j}
$$

(For otherwise we would get $1 \geq 2 a_{j}\left(\left[1 / a_{j}\right]-2\right) \geq 2 a_{j}\left(1 / a_{j}-3\right)>1$ for all $a_{j}$ small enough).

Replacing $v_{j}+s_{j}$ by $v_{j}$ we can assume that $s_{j}=0$. One easily obtains the following inequalities

$$
\int_{\left\{u_{j}<v_{j}+a_{j}^{2}\right\}} \omega_{u_{j}}^{n} \leq 1-\epsilon_{0}, \int_{\left\{v_{j}<u_{j}+a_{j}^{2}\right\}} \omega_{u_{j}}^{n} \leq 1-\epsilon_{0}, \int_{\left\{\left|u_{j}-v_{j}\right|<a_{j}^{2}\right\}} \omega_{u_{j}}^{n} \leq 2 a_{j} .
$$

In [13, Theorem 4.6] we can find $\rho_{j} \in \mathcal{E}(X, \omega)$, such that $\sup _{X} \rho_{j}=0$ and $\omega_{\rho_{j}}^{n}=$ $\frac{1}{1-\epsilon_{0}} 1_{\left\{u_{j}<v_{j}\right\}} \omega_{u_{j}}^{n}+c_{j} 1_{\left\{u_{j} \geq v_{j}\right\}} \omega_{u_{j}}^{n}$ (the constant $c_{j} \geq 0$ is chosen so that the measure has total mass 1 , while $1_{A}$ denotes the characteristic function of the set $A$ ). Set

$$
U_{j}=\left\{\left(1-a_{j}^{3}\right) u_{j}<\left(1-a_{j}^{3}\right) v_{j}+a_{j}^{3} \rho_{j}\right\} \subset\left\{u_{j}<v_{j}\right\} .
$$

By the inequality for mixed Monge-Ampère measures [10] we get

$$
\omega_{u_{j}}^{n-1} \wedge \omega_{\left(1-a_{j}^{3}\right) v_{j}+a_{j}^{3} \rho_{j}} \geq\left(1-a_{j}^{3}\right) \omega_{u_{j}}^{n-1} \wedge \omega_{v_{j}}+\frac{a_{j}^{3}}{\left(1-\epsilon_{0}\right)^{\frac{1}{n}}} \omega_{u_{j}}^{n}
$$

on $U_{j}$. In [11, Theorem 2.3], our assumptions and the definition of $a_{j}$ we obtain

$$
\begin{aligned}
& \left(1-a_{j}^{3}\right) \int_{U_{j}} \omega_{u_{j}}^{n-1} \wedge \omega_{v_{j}}+\frac{a_{j}^{3}}{\left(1-\epsilon_{0}\right)^{\frac{1}{n}}} \int_{U_{j}} \omega_{u_{j}}^{n} \leq \int_{U_{j}} \omega_{\left(1-a_{j}^{3}\right) v_{j}+a_{j}^{3} \rho_{j}} \wedge \omega_{u_{j}}^{n-1} \\
& \leq \int_{U_{j}} \omega_{\left(1-a_{j}^{3}\right) u_{j}} \wedge \omega_{u_{j}}^{n-1}=\left(1-a_{j}^{3}\right) \int_{U_{j}} \omega_{u_{j}}^{n}+a_{j}^{3} \int_{U_{j}} \omega \wedge \omega_{u_{j}}^{n-1} \\
& \leq\left(1-a_{j}^{3}\right)\left(\int_{U_{j}} \omega_{u_{j}}^{n-1} \wedge \omega_{v_{j}}+a_{j}^{4}\right)+a_{j}^{3} \int_{U_{j}} \omega \wedge \omega_{u_{j}}^{n-1} .
\end{aligned}
$$


Hence

$$
\begin{aligned}
& \frac{1}{\left(1-\epsilon_{0}\right)^{\frac{1}{n}}}\left[\int_{\left\{u_{j} \leq v_{j}-a_{j}^{2}\right\}} \omega_{u_{j}}^{n}-\int_{\left\{\rho_{j} \leq-\frac{1-a_{j}^{3}}{a_{j}}\right\}} \omega_{u_{j}}^{n}\right] \leq \frac{1}{\left(1-\epsilon_{0}\right)^{\frac{1}{n}}} \int_{U_{j}} \omega_{u_{j}}^{n} \\
& \leq a_{j}+\int_{U_{j}} \omega \wedge \omega_{u_{j}}^{n-1} \leq a_{j}+\int_{\left\{u_{j}<v_{j}\right\}} \omega \wedge \omega_{u_{j}}^{n-1} .
\end{aligned}
$$

Similarly to $\rho_{j}$ we define $\vartheta_{j} \in \mathcal{E}(X, \omega)$, such that $\sup _{X} \vartheta_{j}=0$ and $\omega_{\vartheta_{j}}^{n}=$ $\frac{1}{1-\epsilon_{0}} 1_{\left\{v_{j}<u_{j}\right\}} \omega_{u_{j}}^{n}+d_{j} 1_{\left\{v_{j} \geq u_{j}\right\}} \omega_{u_{j}}^{n}\left(d_{j}\right.$ plays the same role as $c_{j}$ above). Set

$$
V_{j}=\left\{\left(1-a_{j}^{3}\right) v_{j}<\left(1-a_{j}^{3}\right) u_{j}+a_{j}^{3} \vartheta_{j}\right\} \subset\left\{v_{j}<u_{j}\right\} .
$$

Similarly we get

$$
\begin{aligned}
& \frac{1}{\left(1-\epsilon_{0}\right)^{\frac{1}{n}}}\left[\int_{\left\{v_{j} \leq u_{j}-a_{j}^{2}\right\}} \omega_{u_{j}}^{n}-\int_{\left\{\vartheta_{j} \leq-\frac{1-a_{j}^{3}}{a_{j}}\right\}} \omega_{u_{j}}^{n}\right] \leq \frac{1}{\left(1-\epsilon_{0}\right)^{\frac{1}{n}}} \int_{V_{j}} \omega_{u_{j}}^{n} \\
& \leq a_{j}+\int_{V_{j}} \omega \wedge \omega_{u_{j}}^{n-1} \leq a_{j}+\int_{\left\{v_{j}<u_{j}\right\}} \omega \wedge \omega_{u_{j}}^{n-1} .
\end{aligned}
$$

Note that coupling (3.3), (3.4) and (3.5) one obtains

$$
\begin{aligned}
& \frac{1}{\left(1-\epsilon_{0}\right)^{\frac{1}{n}}}\left[1-2 a_{j}-2 \int_{\left\{\rho_{j} \leq-\frac{1-a_{j}^{3}}{a_{j}}\right\}} \omega_{u_{j}}^{n}\right] \\
& \leq \frac{1}{\left(1-\epsilon_{0}\right)^{\frac{1}{n}}}\left[\int_{\left\{\left|u_{j}-v_{j}\right| \geq a_{j}^{2}\right\}} \omega_{u_{j}}^{n}-2 \int_{\left\{\rho_{j} \leq-\frac{1-a_{j}^{3}}{a_{j}}\right\}} \omega_{u_{j}}^{n}\right] \\
& \leq 2 a_{j}+1 .
\end{aligned}
$$

Now, by Proposition 2.8 and assumption ii), if we let $j \rightarrow \infty$ we would obtain

$$
\frac{1}{\left(1-\epsilon_{0}\right)^{\frac{1}{n}}} \leq 1,
$$

a contradiction.

Thus (3.2) and the whole proof in the bounded functions case is finished.

Below we consider the general case.

Case II: $u_{j}, v_{j} \in \mathcal{E}(X, \omega)$. We can assume, adding constants if necessary, that $\sup _{X} u_{j}=\sup _{X} v_{j}=0$. Choose $t_{j} \rightarrow+\infty$ such that $\lim _{j \rightarrow \infty} \int_{\left\{u_{j} \leq-t_{j}\right\}} \omega_{u_{j}}^{n}+$ $\int_{\left\{v_{j} \leq-t_{j}\right\}} \omega_{v_{j}}^{n}=0$. Using Case I for $\max \left(u_{j},-t_{j}\right)$ and $\max \left(v_{j},-t_{j}\right)$ we obtain $\max \left(u_{j},-t_{j}\right)-\max \left(v_{j},-t_{j}\right) \rightarrow 0$ in $C_{X}$. Therefore $u_{j}-v_{j} \rightarrow 0$ in $C_{X}$. 
Our last result is a generalization from [22, Theorem 5] and from [19, Theorem 3.4]. Kołodziej proved the statement for bounded functions, while Xing needed the additional assumption that $\forall j \geq 1, v_{j} \geq v_{0}$ for some fixed function $v_{0} \in \mathcal{E}(X, \omega)$. Note that such an assumption is quite hard to be checked especially if we know a priori only the Monge-Ampère measures of each $u_{j}$. In fact we show that this condition is superflous.

Theorem 3.8. Let $u_{j}, v_{j}, v \in \mathcal{E}(X, \omega), u \in \operatorname{PSH}(X, \omega)$ and $A>1$ be such that $u_{j} \rightarrow u$ in $L^{1}(X)$ and $\omega_{u_{j}}^{n} \leq A \omega_{v_{j}}^{n}$ for $j \geq 1$. Assume that $v_{j} \rightarrow v$ in $C_{X}$. Then $u \in \mathcal{E}(X, \omega)$ and $u_{j} \rightarrow u$ in $C_{X}$.

Proof. For each $t>0$ we set

$$
\begin{gathered}
u_{j t}:=\max \left(u_{j},-t\right), u_{t}:=\max (u,-t), v_{j t}:=\max \left(v_{j},-t\right), v_{t}:=\max (v,-t), \\
T_{j t}:=\sum_{k=0}^{n-1} \omega_{v_{j t}}^{k} \wedge \omega_{v_{t}}^{n-1-k} .
\end{gathered}
$$

We have

$$
\int_{\left\{u_{j}<u-\delta\right\}} \omega_{u_{j}}^{n} \leq A \int_{\left\{u_{j}<u-\delta\right\}} \omega_{v_{j}}^{n} .
$$

The latter integral can be estimated by

$$
\begin{aligned}
& \int_{\left\{u_{j}<u-\delta\right\}} \omega_{v_{j}}^{n} \leq \int_{\left\{u_{j} \leq-t\right\} \cup\{u \leq-t\} \cup\left\{v_{j} \leq-t\right\}} \omega_{v_{j}}^{n}+\int_{\left\{u_{j t}<u_{t}-\delta\right\}} \omega_{v_{j t}}^{n} \\
& \leq \int_{\left\{u_{j} \leq-t\right\} \cup\{u \leq-t\} \cup\left\{v_{j} \leq-t\right\}} \omega_{v_{j}}^{n}+\frac{1}{\delta} \int_{X}\left|u_{t}-u_{j t}\right| \omega_{v_{j t}}^{n} \\
& \leq \int_{\left\{u_{j} \leq-t\right\} \cup\{u \leq-t\} \cup\left\{v_{j} \leq-t\right\}} \omega_{v_{j}}^{n}+\frac{1}{\delta} \int_{X}\left|u_{t}+\epsilon-u_{j t}\right| \omega_{v_{j t}}^{n}+\frac{\epsilon}{\delta},
\end{aligned}
$$

where we have used from [13, Corollary 1.7] in the first inequality and Chebychev's inequality in the second one.

Note that the middle term can be estimated by

$$
\begin{aligned}
& \frac{1}{\delta} \int_{X}\left(u_{t}-u_{j t}\right) \omega_{v_{j t}}^{n}+\frac{t+\epsilon}{\delta} \int_{\left\{u_{j t}>u_{t}+\epsilon\right\}} \omega_{v_{j t}}^{n}+\frac{\epsilon}{\delta} \\
& \leq \frac{1}{\delta} \int_{X}\left(u_{t}-u_{j t}\right)\left(\omega_{v_{j t}}^{n}-\omega_{v_{t}}^{n}\right)+\frac{1}{\delta} \int_{X}\left(u_{t}-u_{j t}\right) \omega_{v_{t}}^{n} \\
& +\frac{t+\epsilon}{\delta} \int_{\left\{u_{j t}>u_{t}+\epsilon\right\}} \omega_{v_{j t}}^{n}+\frac{\epsilon}{\delta} .
\end{aligned}
$$

Integration by parts in the first term yields (recall the definition of $T_{j t}$ )

$$
\int_{X}\left(u_{t}-u_{j t}\right)\left(\omega_{v_{j t}}^{n}-\omega_{v_{t}}^{n}\right)=\int_{X}\left(v_{t}-v_{j t}\right)\left(\omega_{u_{j t}}-\omega_{u_{t}}\right) \wedge T_{j t} .
$$


Now coupling (3.6) and (3.7) one can estimate $\int_{\left\{u_{j}<u-\delta\right\}} \omega_{v_{j}}^{n}$ by

$$
\begin{aligned}
& \int_{\left\{u_{j} \leq-t\right\} \cup\{u \leq-t\} \cup\left\{v_{j} \leq-t\right\}} \omega_{v_{j}}^{n}+\frac{1}{\delta} \int_{X}\left|v_{t}-v_{j t}\right|\left(\omega_{u_{j t}}+\omega_{u_{t}}\right) \wedge T_{j t} \\
& +\frac{1}{\delta} \int_{X}\left(u_{t}-u_{j t}\right) \omega_{v_{t}}^{n}+\frac{t+\epsilon}{\delta} \int_{\left\{u_{j t}>u_{t}+\epsilon\right\}} \omega_{v_{j t}}^{n}+\frac{2 \epsilon}{\delta}
\end{aligned}
$$

for all $t>0$ and all $\epsilon>0$.

Observe that the second term goes to 0 as $j \rightarrow \infty$, since the integrated function converges to 0 in capacity and all the measures are uniformly absolutely continuous with respect to $C_{X}$ for $j \geq 1$ (and fixed $t$ ). By [7] (recall the argument used in the proof of Theorem 3.4) we also get

$$
\lim _{j \rightarrow \infty} \int_{X}\left(u_{t}-u_{j t}\right) \omega_{v_{t}}^{n}=0
$$

Note that Hartogs' lemma (see [16, Theorem 3.2.13 ]) yields $C_{X}\left(\left\{u_{j t}>u_{t}+\epsilon\right\}\right) \rightarrow$ 0 as $j \rightarrow \infty$. Moreover, since $\omega_{v_{j t}}^{n} \ll C_{X}$ uniformly for $j \geq 1$ we obtain

$$
\lim _{j \rightarrow \infty} \int_{\left\{u_{j t}>u_{t}+\epsilon\right\}} \omega_{v_{j t}}^{n}=0 .
$$

Coupling all these and letting $j \rightarrow \infty$ we get

$$
\varlimsup_{j \rightarrow \infty} \int_{\left\{u_{j}<u-\delta\right\}} \omega_{u_{j}}^{n} \leq \sup _{j \geq 1} \int_{\left\{u_{j} \leq-t\right\} \cup\{u \leq-t\} \cup\left\{v_{j} \leq-t\right\}} \omega_{v_{j}}^{n}+\frac{2 \epsilon}{\delta},
$$

for all $t>0, \epsilon>0$. Letting $\epsilon \rightarrow 0$ and $t \rightarrow+\infty$ we obtain

$$
\lim _{j \rightarrow \infty} \int_{\left\{u_{j}<u-\delta\right\}} \omega_{u_{j}}^{n}=0 .
$$

By Theorem 3.3 we obtain that $u_{j} \rightarrow u$ in $C_{X}$.

\section{References}

[1] S. BenelKourchi, V. Guedu and A. Zeriahi, A priori estimates for weak solutions of complex Monge-Ampère equations, Ann. Sc. Norm. Super. Pisa Cl. Sci. (5) 7 (2008), 81-96.

[2] E. BEDFORD and B. A. TAYLOR, The Dirichlet problem for a complex Monge-Ampère operator, Invent. Math. 37 (1976), 1-44.

[3] E. BEDFORD and B. A. TAYLOR, A new capacity for plurisubharmonic functions, Acta Math. 149 (1982), 1-40.

[4] T. Bloom and N. LeVenberg, Capacity convergence results and applications to a Bernstein-Markov inequality, Trans. Amer. Math. Soc. 351 (1999), 4753-4767. 
[5] U. Cegrell, Pluricomplex energy, Acta Math. 180 (1998), 187-217.

[6] U. CEGRELL, The general definition of the complex Monge-Ampere operator, Ann. Inst. Fourier Grenoble 54 (2004), 159-179.

[7] U. CEgRELL, Convergence in capacity, Technical report, Isaac Newton Institute for Mathematical Sciences, 2001 (arXiv: math/0505218).

[8] U. Cegrell and S. KoŁodzIEJ, The Dirichlet problem for the complex Monge-Ampère operator: Perron classes and rotation invariant measures, Michigan Math. J. 41 (1994), 563-569.

[9] U. Cegrell and S. KołodzIEJ, Equation of complex Monge-Ampère type and stability of solutions, Math. Ann. 334 (2006), 713-729.

[10] S. Dinew, An inequality for mixed Monge-Ampère measures, Math. Z. 262 (2009), 1-15.

[11] S. Dinew, Uniqueness in $\mathcal{E}(X, \omega)$, J. Funct. Anal. 256 (2009), 2113-2122.

[12] V. GUEDJ and A. ZERIAHI, Intrinsic capacities on compact Kähler manifolds, J. Geom. Anal. 15 (2005), 607-639.

[13] V. GUEDJ and A. ZERIAHI, The weighted Monge-Ampère energy of quasiplurisubharmonic functions, J. Funct. Anal. 250 (2007), 442-482.

[14] P. H. HIỆP, On the convergence in capacity on compact Kähler manifolds and its applications, Proc. Amer. Math. Soc. 136 (2008), 2007-2018.

[15] P. H. HIÊP, Convergence in capacity, Ann. Polon. Math. 93 (2008), 91-99.

[16] L. HÖRMANDER, "Notions of Convexity”, Birkhäuser, Boston, 1994.

[17] N. V. KHUE and P. H. HIỆP, A comparison principle for the complex Monge-Ampère operator in Cegrell's classes and applications, Trans. Amer. Math. Soc. 361 (2009), 5539-5554.

[18] S. KotodZIEJ, The Monge-Ampère equation on compact Kähler manifolds, Indiana Univ. Math. J. 52 (2003), 667-686.

[19] S. KotodzIEJ, The set of measures given by bounded solutions of the complex MongeAmpère equation on compact Kähler manifolds, J. London Math. Soc. 124 (2005), 225-238.

[20] S. KoŁodZIEJ, The complex Monge-Ampère equation and pluripotential theory, Memoirs Amer. Math. Soc. 178/840 (2005), 1-64.

[21] Y. XING, Continuity of the complex Monge-Ampere operator, Proc. Amer. Math. Soc. 124 (1996), 457-467.

[22] Y. XING, Continuity of the complex Monge-Ampère operator on compact Kähler manifolds, Math. Z. 263 (2009), 331-344.

Institute of Mathematics Jagiellonian University ul. Łojasiewicza 6 30-348 Kraków, Poland slawomir.dinew@im.uj.edu.pl

Department of Mathematics University of Education (Dai hoc Su Pham Ha Noi) CauGiay, Hanoi, Vietnam phhiep_vn@yahoo.com 